\title{
P003: Current status of infection control practice for prevent of central venous catheter-associated bloodstream infection in Korea
}

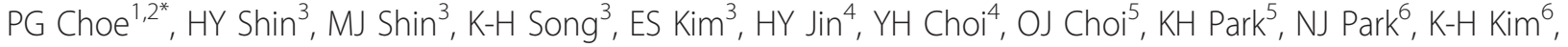 \\ $\mathrm{SH} \mathrm{Han}{ }^{7}$, EJ Choo ${ }^{7}, \mathrm{HB}$ Kim ${ }^{1,3}$, KOrean Study group for Infection Control and prevention (KOSIC) ${ }^{1}$
}

From 2nd International Conference on Prevention and Infection Control (ICPIC 2013)

Geneva, Switzerland. 25-28 June 2013

\section{Introduction}

There are evidence-based guidelines for the prevention of central line-associated bloodstream infections (CLABSI), but the current status of these practices in intensive care units (ICU) of Korea is unknown.

\section{Objectives}

To evaluate the current status of infection control practice for CLA-BSI in ICUs of Korea.

\section{Methods}

We conducted a cross-sectional survey in ICUs of the KOrean Study group for Infection Control and prevention (KOSIC) at April 2012.

\section{Results}

Thirty-five ICUs of 15 hospitals were enrolled in this study. Fourteen of the 35 ICUs (40\%) were medical ICUs, 4 (11\%) were surgical ICUs, 9 (26\%) were neurosurgical ICUs, and 8 (23\%) were combined medical and surgical ICUs. The median bed size was 15 beds (interquartile range [IQR], 14-20), and median patient-tonurse ratio was 1.5 (IQR, 1.3-1.9). During the survey period, the incident rate of CLA-BSI was 3.33 per 1,000 catheter-days (medical ICUs, 5.12; surgical \& neurosurgical ICUs, 1.91; combined ICUs, 2.25).

All ICUs had documented guidelines for the prevention of CLA-BSI and conducted surveillance for CLA-BSI. Nineteen (54\%) ICUs provided regular education programs for CLA-BSI prevention and 15 (43\%) ICUs accessed the adherence to guidelines using a central line

${ }^{1}$ Seoul National University, Seoul, Korea, Republic Of

Full list of author information is available at the end of the article insertion checklist. Twenty-nine (83\%) ICUs used a sterile full body drape during an insertion practice and $3(8 \%)$ ICUs used chlorhexidine preparation with alcohol for an insertion skin preparation. Twenty (57\%) ICUs used antimicrobial-impregnated coated central venous catheter.

All ICUs conducted hand hygiene promotion program including adherence monitoring and 23 (66\%) ICUs conducted active surveillance for multidrug resistant organisms. Hand hygiene adherence was significantly associated with the patient to nurse ratio in ICU $(\gamma=0.648, \mathrm{P}<0.001)$.

\section{Conclusion}

This study demonstrates that although ICUs in Korea had documented guideline and surveillance system for CLS-BSI, infection control practice in real clinic did not meet the recommended practice standard.

\section{Disclosure of interest}

None declared.

\section{Author details}

${ }^{1}$ Seoul National University, Seoul, Korea, Republic Of. ${ }^{2}$ Seoul National University Hosptial, Seoul, Korea, Republic Of. ${ }^{3}$ Seoul National University Bundang Hospital, Seongnam, Korea, Republic Of. ${ }^{4}$ Ajou University Hospital, Suwon, Korea, Republic Of. ${ }^{5}$ Chonnan National University Hospital, Gwangju, Korea, Republic Of. ' ${ }^{6}$ usan National University Hospital, Pusan, Korea, Republic Of. ${ }^{7}$ Soon Cheon Hyang University Bucheon Hospital, Bucheon, Korea, Republic Of.

Published: 20 June 2013

doi:10.1186/2047-2994-2-S1-P3

Cite this article as: Choe et al:: P003: Current status of infection control practice for prevent of central venous catheter-associated bloodstream infection in Korea. Antimicrobial Resistance and Infection Control 2013 2(Suppl 1):P3.

\section{C) Biomed Central}

(c) 2013 Choe et al; licensee BioMed Central Ltd. This is an Open Access article distributed under the terms of the Creative Commons Attribution License (http://creativecommons.org/licenses/by/2.0), which permits unrestricted use, distribution, and reproduction in any medium, provided the original work is properly cited. 